SpyBiotech. It combines the hepatitis B virus surface antigen ( $\mathrm{HBsAg}$ ) with the SpyCatcher/SpyTag 'plug and display' protein conjugation technology to present $S$ protein to the immune system.

HBsAg spontaneously forms a VLP, a structure to which it is possible to attach any protein antigen. The technology, developed by SpyBiotech co-founder Mark Howarth at Oxford University, exploits a domain-the second immunoglobulin-like collagen adhesin domain ( $\mathrm{CnaB} 2)$ - from the Streptococcus pyogenes fibronectin-binding protein $\mathrm{FbaB}$, engineered into a peptide tag (SpyTag) of 13 amino acids and a protein partner (SpyCatcher) of 138 amino acids. When the SpyTag and SpyCatcher come into proximity, a stable intramolecular isopeptide bond spontaneously forms as a result of nucleophilic attack by the unprotonated amine of SpyTag's Lys31 on the carbonyl carbon of SpyCatcher's Asp117 (catalyzed by a neighboring Glu77 residue). The SpyCatcher domain can be embedded in the VLP structure and the SpyTag in any antigen of interest. Mixing the two together results in consistent, stable VLPs that are richly decorated with antigen.

"Making a VLP from scratch with a heterologous antigen does take time," says SpyBiotech co-founder, CEO and CSO Sumi Biswas. But the platform is now in place, and initial clinical data are imminent. "For the next pandemic, you do not have to make the VLP again."

Another VLP technology based on a computationally designed protein nanoparticle developed at the University of Washington's Institute for Protein Design (IPD) is in early stage trials under a partnership between SK Bioscience of Seongnam, South Korea, and CEPI, which have rights to the technology for COVID-19 vaccine development in non-Western markets. Their COVID-19 vaccine, GBP-510, comprises two protein components: a trimer protein to which the target antigen (S protein) is fused and a pentamer that, when combined with the trimer, spontaneously forms a VLP structure. "It can be done in a very controlled way, which is very important for vaccine reproducibility," says CEPI's Jackson. A single particle can accommodate sixty copies of a target antigen. Mouse data show the structure to be highly immunogenic, even at low doses. "We believe the neutralizing titer of a VLP is inherently superior to soluble protein approaches," says Adam Simpson, CEO of Icosavax, which is developing the VLP platform for multiple indications, including COVID-19. "These particles are the proper size for immune trafficking to the lymph nodes."
A group led by the California Institute of Technology's Pamela Bjorkman has reported promising immunogenicity data with mosaic or multivalent structures that employ the SpyCatcher/SpyTag system to decorate the IPD-developed protein nanoparticle. Containing several copies of four to eight different S-protein RBDs from various human and animal coronaviruses, these structures elicited cross-reactive immune responses in mice and provided protection against strains that were not represented in the mosaic. The effects were much stronger than those seen following immunizations with monovalent RBDs or with human convalescent plasma. "It's not that surprising, but it did work really well," says Caltech PhD student Alex Cohen, first author on the paper.

The group has now started testing its construct against a B.1.351 lineage strain. "From our preliminary data, ours doesn't appear to go down very much," says Bjorkman. "The next step will be protection studies." Data from non-human primates will enable the group to benchmark its construct against existing vaccines. That work is getting under way shortly through a collaboration with Malcom Martin at the US National Institute of Allergy and Infectious Diseases. If this approach does yield an effective vaccine, its production would be "trivial" compared with what is entailed in the production of current protein subunit vaccines, Bjorkman says.

Live-attenuated vaccines are, like VLPs, generally highly immunogenic. What's more, they are also expected to elicit an immune response similar to that mounted against an infection, since all of the viral antigens are present. The Serum Institute of India is also at the forefront of this approach, taking forward a live-attenuated COVID19 vaccine through a partnership with Codagenix, which has developed codon deoptimization techniques to impair viral replication. For its SARS-CoV-2 vaccine, COVI-VAC, Codagenix has introduced 283 silent mutations into the gene encoding the viral spike protein. "Our platform is an algorithm-it's not a carrier virus, it's not a VLP," says CEO and co-founder Robert Coleman. Now in phase 1 trials, COVI-VAC is administered as a single-dose, needle-free intranasal vaccine and can be easily manufactured at scale. Although the effective dose has not been established, Coleman estimates it will be in the range of about 1 million plaque-forming units, using standard Vero cell culture production. "That's about fifty doses per milliliter," he says.

Also using a codon-deoptimized live virus is Meissa Vaccines. The company is

\section{Waive COVID-19 IP rights, poor nations urge}

A proposal by the governments of India and South Africa to waive intellectual property protections for COVID-19 vaccines and drugs is being opposed by the US biopharma industry. The proposal, submitted to the World Trade Organization (WTO) Council for Trade-Related Aspects of Intellectual Property Rights (TRIPS) last October, called for IP rights on COVID-19 vaccines and medicines to be suspended, to allow for compulsory licensing until the end of the pandemic crisis. World Health Organization director-general Tedros Adhanom Ghebreyesus said in a speech that he backs the proposal. "The flexibilities in the TRIPS agreement are there to be used in emergencies. If not now, then when?" But a group of developed countries including the United States, United Kingdom, Canada, Japan and Norway, as well as the European Union, blocked WTO consideration of the proposal in December. In a letter to US president Joe Biden on 5 March, Michelle McMurry-Heath, president and CEO of the trade association BIO, called the proposal "both a wrong-headed and ineffective means of spurring further efforts at access." And the lobbying group PHRMA also sent a letter, signed by 30 CEOs, urging the Biden administration to oppose the waiver, saying that eliminating IP protections would undermine the global response to the pandemic. The letter added that COVID-19 vaccine manufacturers are on track to supply 10 billion doses by the end of this year-enough to vaccinate the entire global vaccine-eligible population. Caught in the middle is WTO director-general Ngozi Okonjo-Iweala, who has pressed for a 'third way' that "preserves the multilateral rules that encourage research and innovation while promoting licensing agreements to help scale-up manufacturing of medical products," as she wrote in a Financial Times op-ed on 2 March.

Published online: 12 April 2021 https://doi.org/10.1038/s41587-021-00905-8 